\title{
A Hierarchical Model of Slow Constrained Dynamics
}

\author{
M. A. Muñoz ${ }^{1}$, A. Gabrielli ${ }^{1,2}$, H. Inaoka ${ }^{1,3}$, and L. Pietronero ${ }^{1}$ \\ ${ }^{1}$ Dipartimento di Fisica, Università di Roma "La Sapienza," \\ Piazzale A. Moro 2, I-00185 Rome, Italy \\ ${ }^{2}$ Dipartimento di Fisica, Università di Roma "Tor Vergata" \\ Via della Ricerca Scientifica 1, I-00133 Rome, Italy \\ ${ }^{3}$ Graduate School of Information Sciences, Tohoku University, \\ Sendai 980-77, Japan
}

\begin{abstract}
We introduce a new simple hierarchically constrained model of slow relaxation. The configurational energy has a simple form as there is no coupling among the spins defining the system; the associated stationary distribution is an equilibrium, Gibbsian one. However, due to the presence of hierarchical constraints in the dynamics the system is found to relax to its equilibrium distribution in an extremely slow fashion when suddenly cooled from an initial temperature, $T_{0}$, to a final one $T_{f}$. The relaxation curve in that case can be fit by an stretched exponential curve. On the other hand the relaxation function is found to be exponential when $T_{f}>T_{0}$, with characteristic times depending on both $T_{f}$ and $T_{0}$, with characteristic times obeying an Arrhenius law. Numerical results as well as some analytical studies are presented. In particular we introduce a simple equation that captures the essence of the slow relaxation.
\end{abstract}

PACS number(s): 


\section{INTRODUCTION}

It has been recognized that glasses show many interesting universal properties which have not been satisfactorily explained yet [1,2]. It is well known that glassy states are not ergodic [3]; for example, the diffusive motion of molecules in a liquid near the glass transition temperature becomes much slower than the experimental time scale, and the state of the system is not able to explore the whole phase space within the observation time scale. The relaxation function, $q(t)$, that describes the decay of the system energy (or, analogously, of any other relevant magnitude) towards its stationary state, is observed to evolve in an extremely slow fashion in the vicinity of the glass transition, and is empirically found to be well fit by the Kohlrausch-Williams-Watts or stretched-exponential law, i.e.

$$
q(t)=A \exp \left[-b *(t / \tau)^{\beta}\right]
$$

with exponent $0<\beta<1$ [ [1]. It is often observed that the exponent $\beta$ decreases with decreasing temperature [5].

Nearby the glassy transition standard methods of statistical mechanics based on equilibrium distributions are no longer suitable to describe this kind of systems. A dynamical approach is required for an adequate understanding of slow relaxation processes.

To the date, several dynamical models have been proposed to get some insight into the nature of the slow relaxation processes in glassy dynamics. All of them have in common the presence of some kind of dynamical frustration, and can be classified in two groups: those involving frustration due to the presence of energy and/or entropy barriers (for example spinglass type of models [6 8]), and others in which even with a simple free energy landscape the frustration is directly introduced in the dynamics [10]. The model we present in this paper belongs to the second type, i.e. the energy function is very simple, but the dynamics is strongly constrained; only a reduced number of degrees of freedom can evolve freely at a given time step, while the rest remain frozen.

The new ingredient we include with respect to previously studied models is the presence of a hierarchy of constraints [11]. With that hierarchy we pretend to mimic the fact that in slow relaxation processes, usually there are some degrees of freedom that evolve faster than others and that influence the dynamics of the slower modes in such a way that a whole hierarchy of constrains is generated.

The idea that a theoretical model of slow relaxation should be a dynamical and hierarchically constrained one is not new. It was first introduced by Palmer et. al. [12]. They defined a family of models consisting of discrete levels of degrees of freedom, $n=0,1,2, \ldots$, each level containing $N_{n}$ spins. A spin at level $n+1$ can change freely its state only when $\mu_{n} \leq N_{n}$ spins in level $n$ happen to be at a given specific configuration among all the $2^{\mu_{n}}$ possible ones. As a consequence of such type of dynamical constraints, they argued that a stretched-exponential behavior, Eq.(1), can be reproduced under broad conditions [12]. One important point, however, is that in the previous paper analysis it is implicitly assumed that all the spins above a given one in the chain influence the dynamics of it, and therefore the constraints are long-ranged.

In this paper we consider a very simple microscopic hierarchically constrained model for slow relaxation, which is a particular physical realization of the general scenario introduced in [12], with two important differences: 
1- Our model does not consider long range dynamical constraints, i.e, a degree of freedom is constrained directly only by the nearest degrees of freedom in the hierarchy. As we will show this is enough to generate a slow relaxation.

2- The main advantage of this new model is that the dynamics and the constraints are specifically defined, rendering the model suitable to be analyzed using computer simulations and detailed mathematical analysis.

The paper is structured as follows: in section 2 we introduce the model, in section 3 we present our main numerical results as well as a simple theoretical approximation that reproduces stretched-exponential type of relaxation; we also discuss the nature of the boundary conditions. Finally we present the conclusions. A mathematical formulation of the model and a mean-field like solution are presented in an appendix.

\section{THE MODEL}

The model consists of a set of degrees of freedom (or spins), evolving according to a constrained dynamics. The degrees of freedom could, in principle, be arranged in different ways, but in what follows we consider only a one-dimensional chain with a degree at every site. Each degree of freedom is exposed to the action of an external potential which functional form is schematically shown in Fig.11. It has two local minima at positions $s=0$ and $s=1$ with values 0 and $\epsilon$, respectively, and a maximum of height $\phi>\epsilon$ separating the two minima. We assume that each degree of freedom can be located only at the minima of the potential, and its state can therefore be characterized by a spin-like variable, $s=0,1$.

The spins can change stochastically their state with the following (Arrhenius) transition rates, $\Gamma$

$$
\Gamma_{0 \rightarrow 1}=\tau_{0}^{-1} \exp \left(-\frac{\phi}{T_{f}}\right) \quad, \quad \Gamma_{1 \rightarrow 0}=\tau_{0}^{-1} \exp \left(-\frac{\phi-\epsilon}{T_{f}}\right)
$$

where $\tau_{0}$ is a constant that defines a microscopic time scale, and $T_{f}$ denotes the temperature. Assuming that there is no static interaction among spins, we can easily calculate the equilibrium probability $p_{e q}$ to find a spin at state $s=1$ by using the detailed balance condition:

$$
p_{e q}=\frac{\Gamma_{0 \rightarrow 1}}{\Gamma_{0 \rightarrow 1}+\Gamma_{1 \rightarrow 0}}=\frac{1}{\exp \left(\epsilon / T_{f}\right)+1}
$$

which gives the Gibbs equilibrium distribution associated to the potential in Fig. 1 (observe that it does not depend on $\phi$ ). In the absence of interaction among spins the system exhibits exponential (Debye) relaxation towards the equilibrium distribution with characteristic relaxation time given by: $\tau=p_{p e q} \tau_{0} \exp \left(\phi / T_{f}\right)$.

We now introduce a dynamical constraint in the model: in Fig.2 (a) we show schematically the way in which a spin, $S_{1}$, constrains the motion of its right neighbor $S_{2}$ [13]. When the spin $S_{1}$ is at the state $s_{1}=0$, the dynamics of spin $S_{2}$ is obstructed, i.e., $\phi \rightarrow \infty$, and jumps of $S_{2}$ between the two states are completely prohibited. In the case in which both spins $S_{1}$ and $S_{2}$ are initially at state $s=0$, for $S_{2}$ to jump to the state $s_{2}=1, S_{1}$ has to evolve first to $s_{1}=1$ to clear the path of $S_{2}$. Generalizing this rule the motion of the 
$(i+1)$-th spin in the chain is blocked by the $i$-th spin when this one is at state $s_{i}=0$. Note that while the motion of $s_{i}$ is affected by $s_{i-1}$ it does not depend on $s_{i+1}$, originating in this way a hierarchy of directed constraints. It is important to observe that the dynamics is asymmetric, and that even though there is no static interaction among spins they are dynamically constrained; therefore the equilibrium distribution is the product of the single-site equilibrium distributions eq.(3). This is rigorously proved in the appendix where a probabilistic formulation of the model is presented.

Due to this series of constraints we expect the system to show slow relaxation. One way to see that is in terms of the topology of the phase space: as discussed before, in the two-spin case the system cannot go directly from $\left\{s_{1}, s_{2}\right\}=\{0,0\}$ to $\left\{s_{1}, s_{2}\right\}=\{0,1\}$, but has to take a roundabout path $\{0,0\} \rightarrow\{1,0\} \rightarrow\{1,1\} \rightarrow\{0,1\}$. In the case of $L$-spin system the phase space forms a $L$-dimensional hypercube with sides of size 1 . A vertex of the cube represents a system state. Though the maximum geometrical distance between

any two states is $L^{\frac{1}{2}}$ measured by the edge length of the hypercube, a big fraction of the possible paths are obstructed and unavailable. Therefore the system has to pass through more complex roundabout paths as the system size $L$ increases, and we expect those complex trajectories to give raise to slow relaxation.

\section{RESULTS}

We have performed numerical simulations of the model for different system sizes, $L$, up to $L=1000$, with open boundary conditions [14], i.e. the first spin in the chain (hierarchy) evolves in an unconstrained way (this mimics the fact that typically in glasses there are fast, unconstrained, degrees of freedom). Most of the plots we present are obtained for $L=250$, but the results have been observed to be very robust when increasing system size. Simulations have been carried out for both simultaneous and sequential type of update, and essentially no physical difference is observed among them. Some parameter values are kept fixed in all the simulations: $\tau_{0}=10.0, \phi=1.0$, and $\epsilon=0.5$. We have verified that the qualitative general features exhibited by the model do not depend on the choice of these values. As initial condition the spins are placed at positions $s=0$ or 1 with probabilities corresponding to an equilibrium distribution, $p_{e q}\left(T_{0}\right)$ eq.(3), for a given initial temperature, $T_{0}$. Therefore the dynamics drives the system from an equilibrium distribution at $T_{0}$ to a different equilibrium distribution at $T_{f}$.

We introduce the mean energy per spin defined as

$$
U(t) \equiv L^{-1} \epsilon \sum_{i=1}^{L} s_{i}(t)
$$

(only spins in the $s=1$ state give a non-vanishing contribution to the energy). Given that there is no configurational interaction among spins, after sufficiently long times, $U(t)$ approaches its equilibrium value $U_{e q}=p_{e q} \epsilon$. We define a relaxation function $q(t)$ as

$$
q(t) \equiv\left|U(t)-U_{e q}\right|
$$

which, after a sufficiently long time, $t, q(t)$ approaches zero. 


\section{A. General features of the relaxation curves}

Two typical relaxation curves are shown in figure 4; they correspond to a case in which the system is cooled down, $T_{f}<T_{0}$ (upper one), and in the other the system is heated up, i.e., $T_{f}>T_{0}$. Their behaviors are essentially different.

The uppermost corresponds to $1 / T_{0}=2.19,1 / T_{f}=3$ and $L=250$. Note that the relaxation is very slow; as the time is measured in units of $\tau_{0}=10$, the maximum time corresponds to 15000 time Monte Carlo steps (the curve is the average of $10^{6}$ independent runs). Observe that asymptotically, i.e., after a transient of about $t_{c} \approx 800 \tau_{0}$ time steps the curve is locally well fit by an exponential with a very large characteristic time $\tau \sim 2100 \tau_{0}$, that implies an extremely slow relaxation. However, it is not clear from numerics whether for larger times this exponential behavior will persist, or the curve will decay even in a slower fashion. On the other hand, the whole curve, including the initial faster decay can be perfectly fit by an stretched exponential with $\beta=0.38$ (in fact the fit in undistinguishable from the numerical data in Fig. 田).

Increasing further the time in the computer simulation to decide whether the real asymptotic behavior is an exponential or an stretched exponential is beyond of our available computational power. In any case, for situations in which the system is cooled down, we always get curves that bend progressively in a semilogarithmic plot; and even though the final part can always be fit with a straight line (exponential behavior) in no case it is evident whether that exponential fit gives the right asymptotic behavior. Even for extremely long times we have this type of ambiguity. However, as for any "reasonable" time a stretched exponential can always be fit, we admit the relaxation to be non-exponential is this case. At any rate, the relaxation is extremely slow in the cooling case.

The lower curve in figure 4 corresponds to the same initial temperature, $1 / T_{0}=2.19$, and system size, than the upper one, but a larger final temperature: $1 / T_{f}=1$. In this case the transient is much smaller, $t_{c} \approx 60$, and after it, a pretty clear exponential behavior settles in.

The situation described for the two previous examples is general for all the relaxation curves: those in which $T_{f}<T_{0}$ are well fit by a stretched exponential, while for the opposite situation, $T_{f}>T_{0}$, the decay is exponential after a transient.

We now analyze the transition between the two previous regimes in a more quantitative way. In figure 7 we show the transient time (i.e. the time after which an exponential fit is adequate) as a function of the final temperature for a fixed initial temperature $1 / T_{0}=2.19$.

Note that for very large $T_{f}$ (small $1 / T_{f}$ ) the system can relax very fast, there is no effective frustration and $t_{c}$ is small. In fact, for $1 / T_{f} \rightarrow 0$ the system has a huge degree of thermal activation, and decays exponentially fast to the equilibrium state with no transient. At $T_{f}=T_{0}$ the system is already at equilibrium therefore $t_{c}=0$. Between these two limiting case $t_{c}$ is larger than 0 and behaves in the form shown in Fig. 7; observe that in this interval $t_{c}$ is always relatively small, therefore the asymptotic exponential settles in a short time (smaller than the observation time). On the other hand, when $T_{f}<T_{0}\left(1 / T_{f}>1 / T_{0}\right)$, the behavior is quite different and $t_{c}$ grows fastly without bound. We point out that the previous set of values of $t_{c}$ for $T_{f}<T_{0}$ are obtained fixing some maximum time $(\mathrm{t}=1500)$. If that time is diminished the apparent $t_{c}$ decreases, while it seems to increase for longer times. In this way the values plotted in Fig. 7 are lower bounds for the transient times. In fact, as 
discussed above, if $t_{c}$ converged to a fixed value for $t \rightarrow \infty$, that would imply an exponential asymptotic decay; while a continuously growing $t_{c}$ would imply a stretched exponential type of asymptotic decay. Deciding which of those two possibilities is the right one from numerics is a very difficult task given the extreme slowness of the relaxation process.

Let us now ignore the transients and present a description of the system behavior in terms of its asymptotic decay for the $T_{f}>T_{0}$ case.

For a given pair of fixed initial and final temperatures, the relaxation function is well fit (after the transient) by an exponential (as discussed previously) with a characteristic time $\tau$ that depends on both the initial and the final temperature.

In Fig. 5 and 6 we present the results of our simulations for a chain of length $L=250$ (the results do not change significatively with increasing system size).

In Fig. 5 the dependence of the characteristic time on the final temperature is shown. The curve is well fit by an Arrhenius law: $\tau=C_{1} \exp \left(-C_{2} / T_{f}\right)$ where $C_{1}$ and $C_{2}$ are constants.

In Fig 6 we keep fixed the final temperature, $1 / T_{f}=2$ to study the dependence of the characteristic time on the initial state, we also observe that an Arrhenius law: $\tau=$ $D_{1} \exp \left(-D_{2} / T_{0}\right)$, with $D_{1}$ and $D_{2}$ constants holds. Therefore the characteristic time depends on both the final temperature and the initial state.

We want to point out that in the two previous graphs, if we crossed to the other regime (i.e. $T_{f}<T_{0}$ ), plotting the characteristic time associated to a long time simulation (for example, the one used in figure (7) we would obtain a discontinuous jump: the characteristic time for a slight system cooling $\left(T_{f}=T_{0}-\alpha\right.$, with $\alpha$ small and positive) is much larger (if any) than the one for a slight heating up $\left(T_{f}=T_{0}+\alpha\right)$.

Physically the main difference among the cooling and the heating processes is the following: as can be easily derived from the equilibrium distribution, the average length, $\left\langle l>_{0}\right.$, of chains of spins in the blocking position, $s=0$ is: $\left\langle l>_{0}=1+\exp (\epsilon / T)\right.$. Observe that it goes to 2 at infinite temperature and diverges for vanishing temperature. Analogously the mean length of $s=1$ chains is $\left\langle l>_{1}=1+\exp (-\epsilon / T)\right.$. In order to cool the system down the typical length of a chain of blocking states has to be enlarged. But, of course, the dynamics is restricted to sites preceded by islands of $s=1$ states. As the number and typical length of these islands with $s=1$ is decreased in the cooling process the dynamics becomes slower an slower. On the other hand, when the system is heated up, there is no such an effect: the dynamics is accelerated as more and more active sites appear in the system configuration. In order to render more explicit the previous argument let us perform a mean field type of calculation: as we have seen the total energy of the system is proportional (through $\epsilon$ ) to the number of spins $N_{1}(t)$ in the state 1 . For $N_{1}(t)$ we can write a mean-field master equation:

$$
N_{1}(t+1)=N_{1}(t)+\left(L-N_{1}(t)\right) \eta_{1}(t)-N_{1}(t) \eta_{2}(t)
$$

where $L$ is the system size, $L-N_{1}(t)$ is the number of spins in the state 0 at time $t, \eta_{1}(t)$ is the probability that at time $t$ a spin in the state 0 flips to 1 and $\eta_{2}(t)$ is the probability of flipping from 1 to 0 . If we now divide eq.6 for $L$, we obtain:

$$
\sigma(t+1)=\sigma(t)+(1-\sigma(t)) \eta_{1}(t)-\sigma(t) \eta_{2}(t)
$$

where $\sigma(t)$ is the averaged density of spins at the $s=1$ state at time $t$. In this way, eq. (7) is nothing but an effective balance equation, which establishes that the probability of 
being in $s=1$ at time $t+1$ is given by the probability of being at $s=1$ at time $t$, plus the probability of being at $s=0$ times the probability of jumping from $s=0$ to $s=1$, minus the probability to escape from $s=1$.

We could consider $\eta_{1}$ and $\eta_{2}$ as given by some fixed values, but in order to keep more track of the original constrained nature of the dynamics dynamics we choose them in a slightly different way: as $\sigma(t)$ can be interpreted as the probability that a fixed spin is in the state $s=1$ at time $t$, we take $\eta_{1}=0$ with probability $1-\sigma(t)$, that is the transition probability is 0 if the preceding spin is in a blocking state, and $\eta_{1}=\exp \left(-1 / T_{f}\right)$ (we have taken $\phi=\tau_{0}=1$ ) with complementary probability $\sigma(t)$, according to eq. (目). Analogously, $\eta_{2}=0$ with probability $1-\sigma(t), \eta_{2}=\exp \left((\epsilon-1) / T_{f}\right)$ with probability $\sigma(t)$.

In this way, as the system relaxes to its stationary state, the transition probabilities change and modify the rate at which the dynamical variable $\sigma(t)$ evolves.

The previous simple (but not trivial) equation can not be solved analytically, but it is simple to iterate numerically. We have considered $\sigma(t=0)$ given by its equilibrium value at some temperature $T_{0}$, and iterate the equation for different values of the temperature $T_{f}$. The result of such an analysis are shown in figure 8. For any set of parameter values the resulting relaxation curve can be fit by an stretched exponential with exponent $\beta<1$. We will further study the curious analitycal properties of this simple approach in a future work.

Therefore, even in this simple approach, in which the spatial structure is almost completely disregarded, we reproduce a stretched-exponential type of decay, due to the fact that the jumping probability decays in time as the number of blocking states grows.

\section{B. Open versus periodic boundary conditions}

A possible way to synthesize a stretched-exponential is by the convolution of a number of exponential curves with different characteristic times, $\tau_{n}$, i.e.

$$
q(t)=\sum_{n} w_{n} \exp \left(-t / \tau_{n}\right)
$$

where $w_{n}$ are some weight factor.

In order to shed some light on the microscopic origin of that slow relaxation process, and clarify whether the stretched-exponential behavior describing the transient behavior of the relaxation function comes from a convolution like that of Eq.(8) (with the $n$ in the sum being the position in the chain), we have studied the time relaxation of the energy

of the spin at every site as a function of its position in the chain. In Fig.9 we show the time evolution of $U(i, t)-U_{e q}$, with $U(i, t)=\epsilon\left\langle s_{i}(t)\right\rangle$, where $\langle\ldots\rangle$ stands for averages over different runs. It is observed that the first spin in the chain relaxes faster than the second one, the second one faster than the third, and so on. I fact, the energies of the first spins $U(i, t)$ relax exponentially fast to its equilibrium value, $U_{e q}=p_{e q} \epsilon$, with a time constant, $\tau_{i}$, that increases with the position $i$ in the chain. After a certain number of spins that depends on the parameter values, the relaxation of every single spin is indistinguishable from the relaxation observed performing a simulation with periodic boundary conditions and the same parameter values: this is what we call the bulk behavior. Therefore, the only difference between periodic and open boundary conditions is a small effect that does not affect the bulk properties. As apparent stretched-exponential behavior are observed for the 
relaxation of the bulk spins when $T_{f}<T_{0}$, we conclude that it is not due to a convolution of exponential functions with different characteristic times associated to the different positions in the chain.

An alternative possible way to understand the apparent stretched exponential behavior as associated to a convolution of exponentials with different characteristic times is by assuming that the islands of spins in position $s=1$ in the system relax in a different way depending on how many blocking spins are placed in the immediately superior positions in the chain. In this way it is clear that spins blocked by only one spin will relax much faster than spins preceded by long chains of blocking spins. This mechanism would give rise to different relaxation times for spins in different relative positions in the chain, and therefore to a global stretched-exponential behavior.

\section{CONCLUSIONS}

We have presented a simple model of slow relaxation. Its stationary equilibrium distribution is a simple Gibbs distribution, but its dynamics is strongly constrained. That gives rise to slow relaxation processes in the case in which the system is cooled from an initial temperature, $T_{0}$ to a smaller $T_{f}$. The asymptotic behavior of the relaxation function, after a transient, is found to be exponential when heating the system up, with a relaxation time depends on both the initial and the final temperatures in a non-trivial way. When cooling the system down it is not clear whether the relaxation function reaches an exponential behavior asymptotically or if it is a stretched-exponential even asymptotically. In any case, for small enough final temperature, and for large initial temperatures, the system is found to show a extremely slow relaxation originated by the constrained dynamical rules. In this way we put forward, using a very simple model, how constrained dynamics can slow down relaxation processes in quite a dramatic way. We have also introduced a very simple one-variable equation that captures the essence of the slow relaxation and reproduces stretched-exponential type of decay.

\section{ACKNOLEDGEMENTS}

We thanks C. A. Angell, U. Marini Bettollo, Sebastiano Carpi, and G. Parisi for useful discussions. We are grateful to the anonymous referee whose comments and criticisms helped us to improve notoriously this paper. This work was partially supported by the Japan Society

for the Promotion of Science through a grant to H.I. and by the European Union through a grant to M.A.M.

\section{APPENDIX: ANALYTICAL APPROACH}

Now we present an analytical attempt to understand the previously described properties. Our model can be formally represented in terms of a Markov chain, defined by the following equation:

$$
P(\{s\} ; t)=\sum_{\left\{s^{\prime}\right\}} W\left(\left\{s^{\prime}\right\} \rightarrow\{s\}\right) P\left(\left\{s^{\prime}\right\} ; t-1\right)
$$


where $\{s\}=\left\{s_{i}=s_{i}(t)\right\}$, and $\left\{s^{\prime}\right\}=\left\{s_{i}^{\prime}=s_{i}(t-1)\right\}$ for every $i=1,2, \ldots, L$, are system configurations at time $t$ and $t-1$ respectively. The equation 9 states how the probability, $P(\{s\} ; t)$ of finding the system at a given time $t$ in a configuration $\{s\}$ evolves in time. The transition probabilities, $W\left(\left\{s^{\prime}\right\} \rightarrow\{s\}\right) P\left(\left\{s^{\prime}\right\} ; t-1\right)$ are given by:

$$
W\left(\left\{s^{\prime}\right\} \rightarrow\{s\}\right)=\prod_{i=1}^{L} \omega\left(s_{i}^{\prime} \rightarrow s_{i} ; s_{i-1}^{\prime}\right)
$$

with

$$
\omega\left(s_{i}^{\prime} \rightarrow s_{i} ; s^{\prime}{ }_{i-1}\right)=\delta_{s^{\prime}, s_{i}}-\delta_{s^{\prime}{ }_{i-1}, 1}(-1)^{s^{\prime}{ }_{i}+s_{i}} \frac{\exp \left(\beta \epsilon s_{i}^{\prime}\right)}{\tau_{0} \exp (\beta \phi)}
$$

and the boundary condition $s_{0}(t)=1$. This is nothing but the mathematical expression of the transition probabilities described in the previous section.

By direct substitution it is easy to verify that the equilibrium distribution

$$
P(\{s\} ; e q)=\prod_{i=1}^{L} \frac{\exp \left(-\beta \epsilon s_{i}\right)}{1+\exp (-\beta \epsilon)}
$$

is the stationary solution of Eq.(9).

From the general equation Eq.(9), we can derive a hierarchy of equations for the $m$ body probability distributions (similar to the BBGKY hierarchy in statistical mechanics), In particular, for $m=1$

$$
p\left(s_{i} ; t\right)=\sum_{s^{\prime}{ }_{i-1}=0}^{1} \sum_{s^{\prime}{ }_{i}=0}^{1} \omega\left(s_{i}^{\prime} \rightarrow s_{i} ; s_{i-1}^{\prime}\right) p\left(s_{i-1}^{\prime}, s_{i}^{\prime} ; t-1\right)
$$

where

$$
p\left(s_{i} ; t\right)=\sum_{s_{1}=0}^{1} \ldots \sum_{s_{i-1}=0}^{1} \sum_{s_{i+1}=0}^{1} \ldots \sum_{s_{L}=0}^{1} P(\{s\} ; t)
$$

are the one-body probability functions at time $t$,

$$
p\left(s_{i-1}^{\prime}, s_{i}^{\prime} ; t-1\right)=\sum_{s^{\prime}{ }_{1}=0}^{1} \ldots \sum_{s^{\prime}{ }_{i-2}=0}^{1} \sum_{s^{\prime}{ }_{i+1}=0}^{1} \ldots \sum_{s^{\prime}{ }_{L}=0}^{1} P\left(\left\{s^{\prime}\right\} ; t-1\right)
$$

are the two-body probability functions at time $t-1$. Analyzing this set of equations is a difficult task as can be seen from the fact that the one-body probability functions depend on the two-body probabilities distributions $p\left(s_{i-1}, s_{i} ; t-1\right)$; the equations for these depend on three-body probabilities and so on.

The first analytical approach ( the only one we complete in this paper) consists of approximating the two-body probabilities by the product of two one-body probability functions, namely:

$$
p\left(s_{i-1}^{\prime}, s_{i}^{\prime} ; t-1\right) \approx p\left(s^{\prime}{ }_{i-1} ; t-1\right) p\left(s_{i}^{\prime} ; t-1\right)
$$


This is a mean field like approximation given that high order correlation are neglected. Introducing Eq.(16) in Eq.(13), and after some simple algebra, we get

$$
p_{i}(t)-p_{i}(t-1)=\frac{1}{\tau_{0} \exp (\beta \phi)}\left[p_{i-1}(t-1)\left(1-p_{i}(t-1)\right)-\exp (\beta \epsilon) p_{i-1}(t-1) p_{i}(t-1)\right]
$$

where $p_{i}(t)=p\left(s_{i}=1 ; t\right)$. In the case of an open chain we put $p_{0}(t)=1$, which corresponds to the fact that the first spin is unconstrained at any time.

In the continuous time limit, i.e. when $\tau_{0} \gg 1$ (which is the case in the numerical simulations), the previous equation can be written as:

$$
\frac{d p_{i}(t)}{d t}=\frac{1}{\tau_{0} \exp (\beta \phi)}\left[p_{i-1}(t)\left(1-p_{i}(t)\right)-\exp (\beta \epsilon) p_{i-1}(t) p_{i}(t)\right]
$$

which general solution is

$$
p_{i}(t)=p_{e q}+\left(p_{i}(0)-p_{e q}\right) \exp \left(I_{i}(t) / \tau_{1}\right),
$$

with $\tau_{1}=\tau_{0} p_{e q} \exp \left(\phi / T_{f}\right)$ (which is the free relaxation time) and $I_{i}=\int_{0}^{t} p_{i-1}\left(t^{\prime}\right) d t^{\prime}$ is an effective time for $i$-th spin. Substituting $I_{1}=t$ in Eq.(19), we can calculate $I_{2}$. It is obtained to be given asymptotically by $I_{2} \simeq p_{e q} t$ for $t \gg \tau / p_{\text {eq }}$, and therefore Eq.(19), for $i=2$ can be rewritten as

$$
p_{i}(t)=p_{e q}+\left(p_{i}(0)-p_{e q}\right) \exp \left(-p_{e q} t / \tau_{1}\right)
$$

By iterating the same procedure we get $I_{i} \simeq p_{e q} t$ and Eq.(20) as the solution for $p_{i}$ for any $i>1$. The relaxation time for $i$-th spin $\tau_{i}$ by Eq.(20) is

$$
\tau_{i}=\tau_{1} / p_{e q} \sim \exp \left(\text { const. } \times \frac{1}{T_{f}}\right)
$$

which has a temperature dependence as that of strong liquids. However, this mean field approximation does not reproduce correctly the characteristic decay times. We expect that more accurate approximations to Eq.(9) reproduce more accurately the behavior of the relaxation function. Results obtained by truncating the hierarchy at higher levels will be presented elsewhere [15]. 


\section{REFERENCES}

[1] C. A. Angell, Science 267, 1924 (1995) and references there in.

[2] Complex behaviour of Glassy Systems, Ed. M. Rubí and C. Pérez-Vicente, Lecture Notes in Physics, 492, 1997.

[3] R. G. Palmer, Adv. Phys. 31, 669 (1982).

[4] R. Böhmer, K. L. Ngai, C. A. Angell, D. J. Plazek, J. Chem. Phys. 99, 4201 (1993).

[5] P. K. Dixon and S. R. Nagel, Phys. Rev. Lett. 61, 341 (1988).

[6] S. Franz and G. Parisi, J. Phys. I France, 5, 1401 (1995).

[7] F. Ritort, Phys. Rev. Lett. 75, 1190 (1995).

[8] J. P. Bouchaud and M. Mezard, J. Phys. I France, 4, 1109 (1994)

[9] G. W Evans, Rev. Mod. Phys. 65, 1281 (1993).

[10] E. Follana and F. Ritort, Phys. Rev. B 54, 930 (1996).

[11] Models that present some resemblance with the one we introduce here have been previously studied in a different context; see for instance, G. W Evans, Rev. Mod. Phys. 65 , 1281 (1993).

[12] R. G. Palmer, D. L. Stein, E. Abrahams, and P. W. Anderson, Phys. Rev. Lett. 53, 958 (1984).

[13] L. Pietronero, in Fractals in Physics, edited by L. Pietronero and E. Tosatti (Elsevier Science Publishers B. V., 1986), pp. 417.

[14] Note that with periodic boundary conditions the system could reach an absorbing state, $s_{i}=0$, for all $i$, from which the system cannot escape.

[15] A. Gabrielli, M.A. Muñoz and L. Pietronero, in preparation. 


\section{FIGURES}

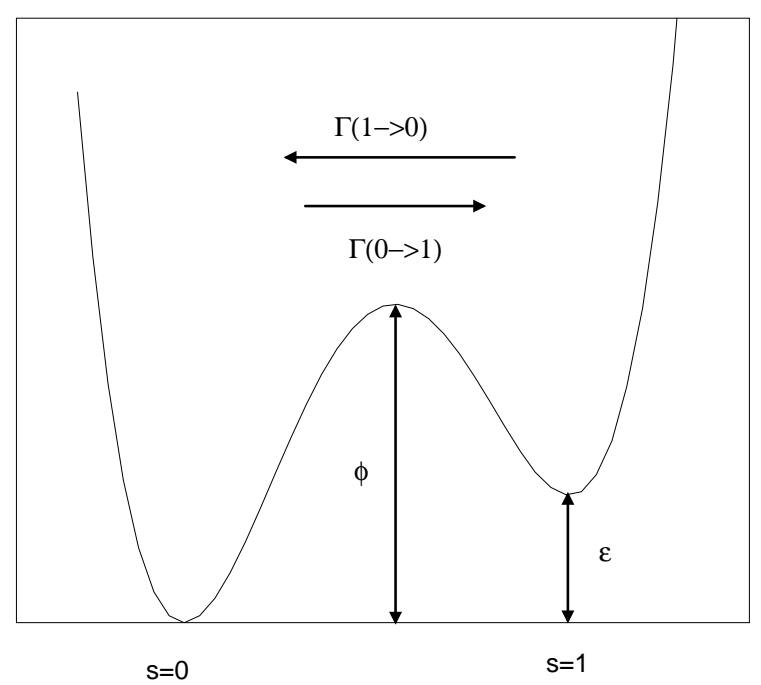

FIG. 1. A schematic figure of the potential form in which atoms are placed in our model. Two local minima $s=0$ and 1 are separated by a maximum of height $\phi$.

FIG. 2. When the spin 1 is placed at position $s_{1}=0$ the transition of the spin 2 is prohibited.

FIG. 3. The phase space for the two spin system. The direct path from $\left\{s_{1}, s_{2}\right\}=\{0,0\}$ to $\{0,1\}$ is obstructed and the transition between these two states is prohibited. 


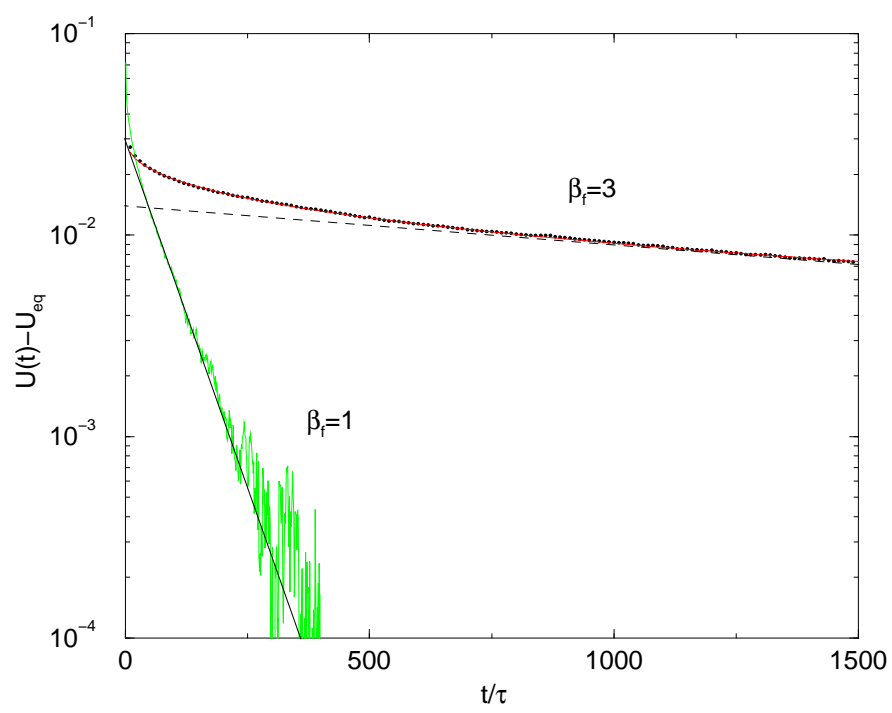

FIG. 4. Typical relaxation curves for $T_{f}<T_{0}$ (uppermost, $1 / T_{f}=3$ and $1 / T_{0}=2.19$ ), and $T_{f}>T_{0}$ (lowermost $1 / T_{f}=1$ and $\left.1 / T_{0}=2.19\right)$. The second one is exponential while the first one can be fit by an stretched exponential with $\beta=0.38$; an exponential fit (dashed line) is valid for times larger than $\approx 800 . L=250$.

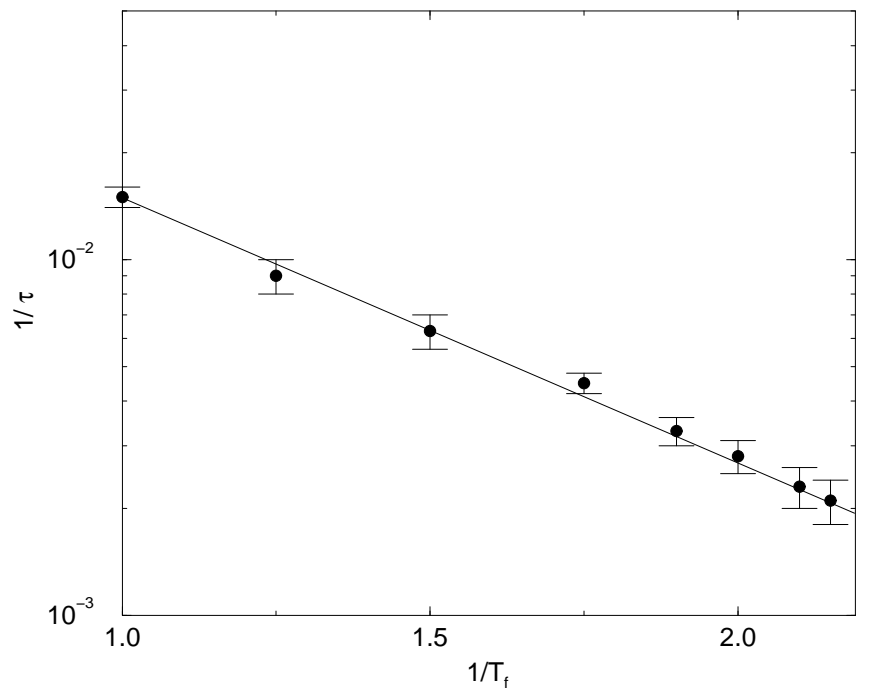

FIG. 5. Semilog plot of the inverse of the characteristic time as a function of $1 / T_{f}$ for a fixed $1 / T_{0}=2.19 . \quad L=250$. 


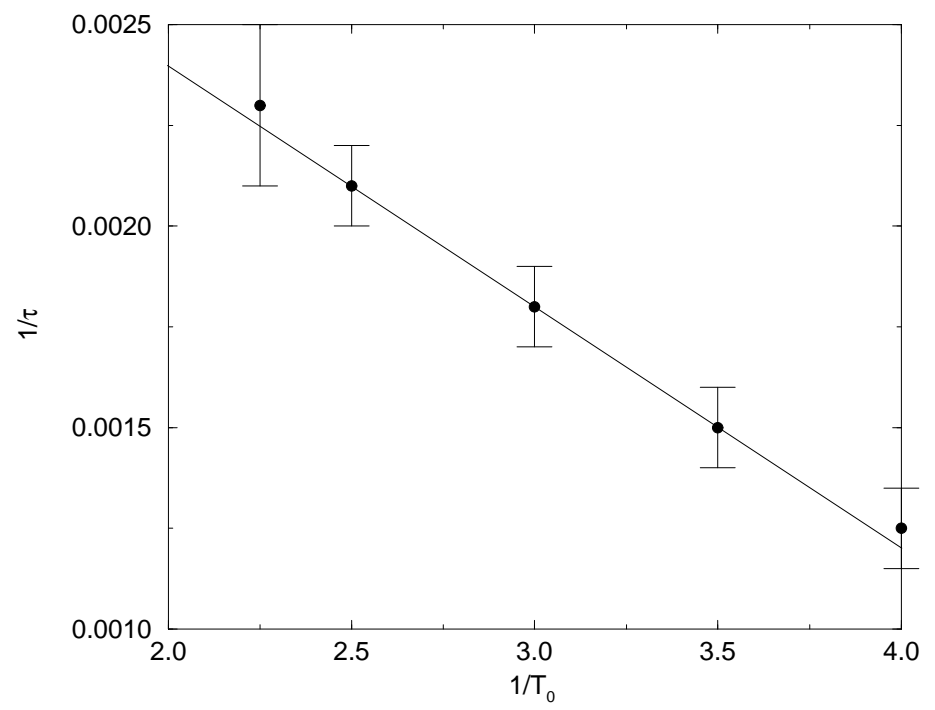

FIG. 6. Semilog plot of the inverse of the characteristic time as a function of $1 / T_{0}$ for a fixed $1 / T_{f}=2 . L=250$.

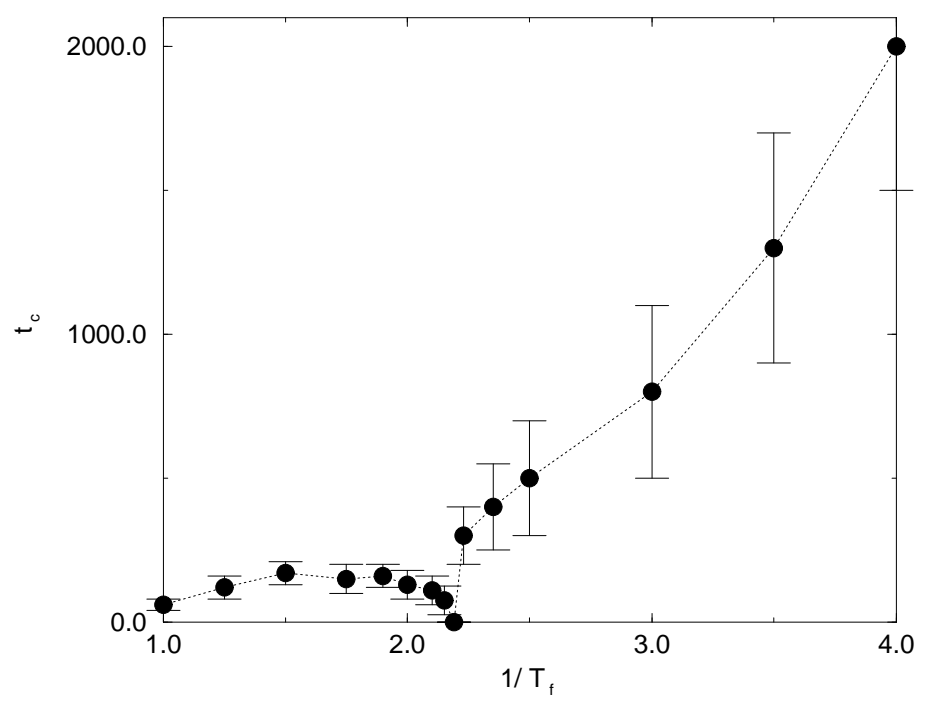

FIG. 7. Transient time (as defined in the text) as a function of $1 / T_{f}$, with $1 / T_{0}=2.19$. $L=250$. 


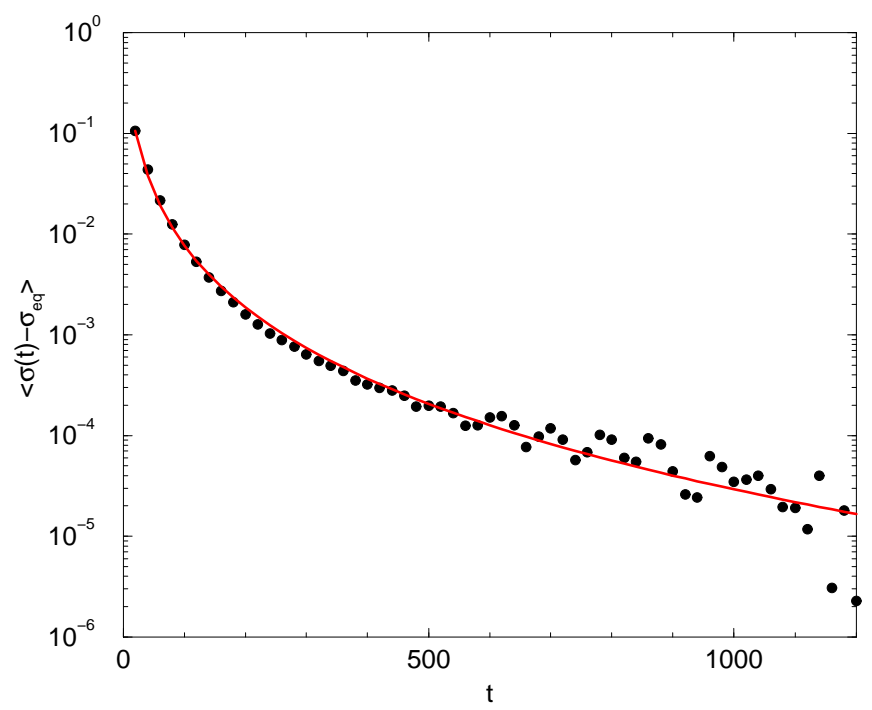

FIG. 8. Stretched exponential decay associated to eq.(7), when the system relaxes with $1 / T_{f}=3$ from an initial $\sigma(0)=1 /(1+\exp (1 / 2))$. The fit is given by $b=3.7535$ and $\beta=0.201$ (see eq.(1)).

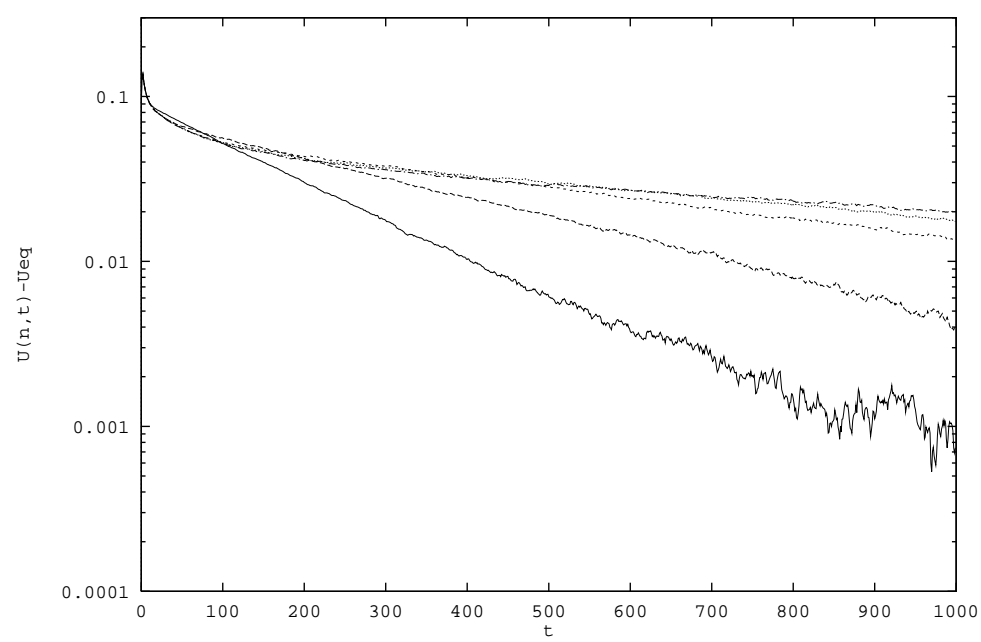

FIG. 9. Semilog plot of the decay of $U(i, t)-U_{e q}$ for the first seven spins in an open chain as a function of time, for a system with $L=250$. The lowermost curve corresponds to the first spin in the chain, the next one to the second one and so on. 


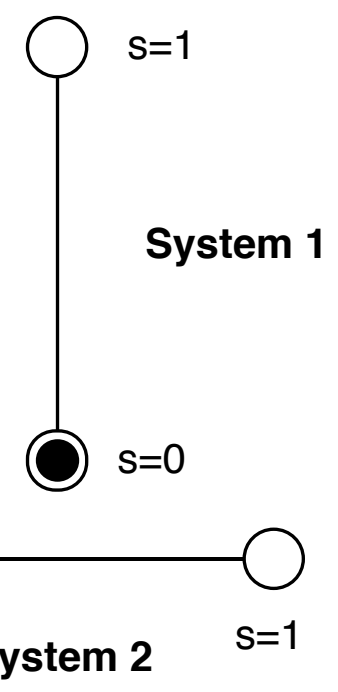




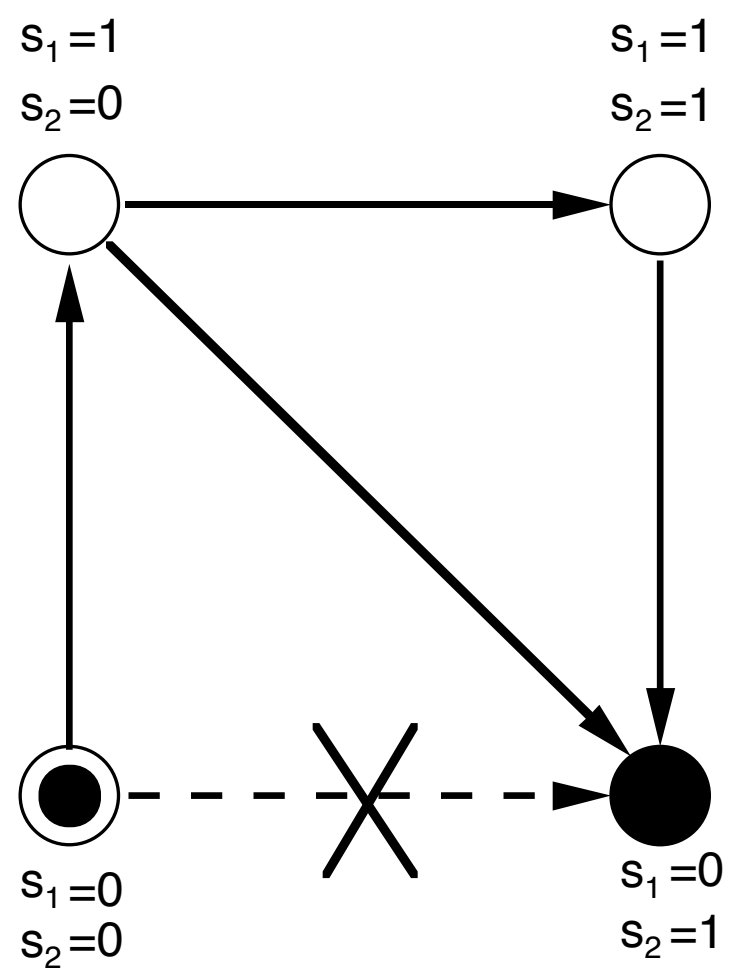

